\title{
Educação, Saúde Pública e Teoria da Comunicação: o curso de pós- graduação em Comunicação e Saúde da ESP-MG
}

\author{
Education, Public Health and Communication Theory: the specialization \\ course in Communication and Health of ESP-MG
}
Educación, Salud Pública y Teoría de la Comunicación: el curso de especialización en Comunicación y Salud de la ESP-MG

Cláudia Chaves Fonseca ${ }^{1, a}$

claufon@gmail.com | http://orcid.org/oooo-0002-7010-7415

Jean Alves de Souza ${ }^{1, b}$

jean.espmg@yahoo.com.br | http://orcid.org/o0oo-0002-4486-7963

${ }^{1}$ Escola de Saúde Pública de Minas Gerais. Minas Gerais, MG, Brasil.

a Doutorado em Educação pela Pontifícia Universidade Católica de Minas Gerais.

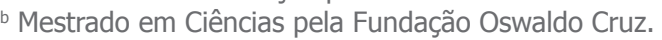

\section{Resumo}

A interface entre a comunicação e a saúde é construída coletivamente, envolvendo uma miríade de interlocutores em contextos sociais determinados, com o objetivo final de tornar efetivos os direitos à comunicação e à saúde, previstos na Constituição Federal de 1988. Para promover este encontro, bem como capacitar profissionais de ambas as áreas, a Escola de Saúde Pública de Minas Gerais (ESP-MG) oferece o curso de Pós-graduação em Comunicação e Saúde, no qual a disciplina Teorias da Comunicação tem destaque, uma vez que é primordial conhecer os fundamentos da comunicação humana e a dinâmica da Comunicação Social, com ênfase no campo teórico-prático conhecido como Comunicação e Saúde (CeS). Este artigo relata a experiência do curso a partir da disciplina Teorias da Comunicação, de modo a situá-la como conhecimento essencial não somente para os comunicadores, mas também para os profissionais de saúde.

Palavras-chave: Comunicação; Educação; Saúde pública; Teorias da comunicação; Pós-graduação. 


\section{Abstract}

The interface between communication and health is built collectively, involving a myriad of interlocutors in determined social contexts, with the ultimate goal of making the communication and health rights established in the 1988 Federal Constitution effective. In order to promote this exchange, as well as training professionals from both areas, the School of Public Health of Minas Gerais (SP-MG) offers the postgraduate course in Communication and Health, in which the Theories of Communication discipline is highlighted, since it is essential to know the fundamentals of human communication and the dynamics of Social Communication, with emphasis on the theoretical-practical field known as Communication and Health (CES). This article reports the experience of the course from the Theories of Communication subject, in order to situate it as essential knowledge not only for communicators, but also for health professionals.

Keywords: Communication; Education; Public health; Communication theories; Postgraduate studies.

\section{Resumen}

La interfaz entre la comunicación y la salud se construye colectivamente, involucrando una miríada de interlocutores en contextos sociales determinados, con el objetivo final de hacer efectivos los derechos a la comunicación ya las saludes previstas en la Constitución Federal de 1988. Para promover este encuentro, como la capacitación de profesionales de ambas áreas, la Escuela de Salud Pública de Minas Gerais (ESP-MG) ofrece el curso de Postgrado en Comunicación y Salud, en el cual la disciplina Teorías de la Comunicación tiene destaque, ya que es fundamental conocer los fundamentos de la comunicación humana y la dinámica de la Comunicación Social, con énfasis en el campo teórico-práctico conocido como Comunicación y Salud (CeS). Este artículo relata la experiencia del curso a partir de la disciplina Teorías de la Comunicación, para situarla como conocimiento esencial no sólo para los comunicadores, sino también para los profesionales de la salud.

Palabras clave: Comunicación; La educación; Salud pública; Teorías de la comunicación; Posgraduación.

Contribuição dos autores:

Concepção e desenho do estudo: Cláudia Chaves Fonseca.

Aquisição, análise ou interpretação dos dados: Jean Alves de Souza.

Redação do manuscrito: Cláudia Chaves Fonseca, Jean Alves de Souza.

Revisão crítica do conteúdo intelectual: Cláudia Chaves Fonseca, Jean Alves de Souza.

Declaração de conflito de interesses: não há.

Fontes de financiamento: não houve.

Considerações éticas: não há.

Agradecimentos/Contribuições adicionais: não há.

Histórico do artigo: submetido: 24 jul. 2019 | aceito: 27 fev. 2019 | publicado: 30 jun. 2020.

Apresentação anterior: não houve.

Licença CC BY-NC atribuição não comercial. Com essa licença é permitido acessar, baixar (download), copiar, imprimir, compartilhar, reutilizar e distribuir os artigos, desde que para uso não comercial e com a citação da fonte, conferindo os devidos créditos de autoria e menção à Reciis. Nesses casos, nenhuma permissão é necessária por parte dos autores ou dos editores. 


\section{Introdução}

A comunicação humana é um processo social básico e preliminarmente se refere ao conjunto das interações em um meio social. Quando nos comunicamos, não somente nomeamos os objetos do mundo, mas partilhamos nossa subjetividade, trocamos ideias, informações; bem como atribuímos sentido e valor às diferentes situações. O desenho, o alcance, os recursos, as estratégias e as finalidades das interações, em espaços e tempos determinados, compõem um formidável leque de questões para pesquisa e investigação. Tal fenômeno constitui um campo disciplinar e acadêmico de reflexão - conhecido como Teorias da Comunicação - que se desenvolveu desde o início do século passado e que não se pode mais desconhecer, conforme nos adverte Braga: “(...) O que parece importar é a constatação inarredável, na presente situação históricosocial, da objetivação de um espaço de estudos, reflexões e pesquisa percebidos largamente como relevantes, espaço este que, ao ser nomeado pelo termo 'comunicação' ou pela expressão 'comunicação social', encontra forte consenso quanto ao de que se está falando - ainda que o contorno e a organização interna desse espaço estejam longe de ser consensuais"1 (p. 11).

Qual seria, então, o objeto preciso a ser estudado? Se conceituarmos 'comunicação' como uma variedade de processos de conversação social, de trocas simbólicas generalizadas, infere-se que vários campos do conhecimento são implicados, a exemplo da Sociologia, da História, da Antropologia, da Psicologia etc. Para que a definição do objeto não se disperse em uma amplitude vaga, é prudente ter em mente que: “(...) Assim, nas demais áreas do conhecimento, ou a comunicação é observada sem ser problematizada; ou então é problematizada em função de interesses específicos da área. No campo da comunicação, todo e qualquer fato humano será problematizado no ângulo comunicacional"1 (p. 18).

Por outro lado, adotar uma perspectiva oposta, definindo como objeto de estudo da Comunicação somente os meios de comunicação coletiva é reduzir drasticamente a concepção, uma vez que a comunicação abrange tanto as interações face a face quanto aquelas entre grupos, entre as empresas e seus públicos, entre o governo e a sociedade etc. Por esta razão, Braga ${ }^{1}$ afirma a importância de um olhar, de um ângulo comunicacional que dirija a problematização. Desse modo, a comunicação será compreendida em sua dimensão relacional e assim estudada.

Ao seguir o raciocínio, observamos muitas vezes que o campo da Comunicação faz interface com outras áreas do saber, entre elas cita-se a Saúde Pública. Neste caso, há pelo menos duas décadas vem sendo constituída, no meio acadêmico e na prática social, uma interface denominada Comunicação e Saúde (CeS). O objetivo deste artigo é refletir sobre o estudo da Teoria da Comunicação, mais especificamente a disciplina de mesmo nome, em um curso de pós-graduação em Comunicação e Saúde Pública, voltado a profissionais de ambas as áreas. Pergunta-se: de que modo a perspectiva comunicacional pode ajudar o profissional de saúde ou o profissional de comunicação em saúde a qualificar sua atuação? Quais são as contribuições esperadas?

\section{O campo da Comunicação e Saúde}

A CeS é um campo interdisciplinar para o qual convergem as teorias, os recursos e as práticas dessas áreas. Na sociedade complexa e digitalizada em que vivemos, o tema saúde não diz mais respeito somente à conservação e à ampliação da vida biológica envolvendo processos de prevenção e cura de doenças; mas, ao fazê-lo, põe em movimento um aparato de interesses econômicos, políticos, de gestão social etc. O campo Comunicação e Saúde busca refletir a respeito deles: "O termo Comunicação e Saúde, portanto, delimita um território de disputas específicas, embora atravessado e composto por elementos característicos de um, de outro e da formação social mais ampla que os abriga. Trata-se de um campo ainda em formação, mas como os demais constitui um universo multidimensional no qual agentes e instituições desenvolvem estratégias, tecem alianças, antagonismos, 
negociações. Essa concepção implica colocar em relevo a existência de discursos concorrentes, constituídos por e constituintes de relações de saber e poder, dinâmica que inclui os diferentes enfoques teóricos acerca da comunicação, saúde e suas relações. Contrapõe-se, assim, a perspectivas que reduzem a comunicação a um conjunto de técnicas e meios a serem utilizados de acordo com os objetivos da área da saúde, notadamente para transmitir informações de saúde para a população”2 (p. 31).

Como afirmam as autoras, este é um campo teórico-prático ainda em construção. É importante ressaltar este ponto porque, , por muito tempo a comunicação foi vista pelos profissionais de saúde como algo que se aciona em caso de necessidade, como nas campanhas preventivas. Atualmente, o raciocínio já se desenvolveu no sentido de conceber a comunicação como uma mediação construída pelos vários envolvidos: comunicadores, profissionais de saúde, público, gestores etc.

Busca-se, por meio da Comunicação e Saúde, construir uma conversação possível, de modo que as interações (entre elas uma que 'joga' com uma importância fundamental: a abordagem midiática) tratem com equidade os saberes entre os diversos agentes e instituições envolvidos com a saúde (profissionais, hospitais e clínicas, indústrias, mídia, assessores de comunicação, público, entre outros). Parte-se do princípio de que possam ser construídos interesses comuns, um saber existente a ser compartilhado e outros saberes a serem (re)construídos em uma perspectiva coletiva. É uma perspectiva na qual os campos da comunicação e saúde não têm relação de subalternidade: a comunicação e saúde não são vistos de forma que uma seja hierarquicamente superior à outra, mas de forma equivalente e complementar.

A disciplina Teorias da Comunicação, ministrada no curso de especialização em Comunicação e Saúde da ESP-MG, é guiada por essa concepção. A seguir são apresentadas a Escola e o planejamento do curso.

\section{A Escola de Saúde Pública de Minas Gerais (ESP-MG)}

A Escola de Saúde Pública do Estado de Minas Gerais (ESP-MG) foi criada em 3 de junho de 1946, pelo Decreto $\mathrm{n}^{\mathrm{o}} 1.751 / 1946^{3}$, em meio ao processo de reestruturação do Departamento de Higiene do Estado de Minas Gerais, sendo a primeira Escola de Saúde Pública estadual no Brasil. Atualmente é um órgão autônomo da administração direta estadual, vinculada tecnicamente à Secretaria de Estado de Saúde de Minas Gerais (SES-MG) e, junto à Fundação Hospitalar do Estado de Minas Gerais (FHEMIG), à Fundação Ezequiel Dias (Funed) e à Fundação Centro de Hematologia e Hemoterapia de Minas Gerais (Hemominas), formam o Sistema Estadual de Saúde de Minas Gerais.

As diretrizes projetadas na época da criação da ESP-MG ainda permanecem: especializar em áreas da saúde pública profissionais graduados atuantes na área de saúde; formar profissionais técnicos e auxiliares nas áreas de saúde pública; referenciar-se pelas demandas dos serviços de saúde; integrar o ensino e o trabalho dos profissionais de saúde e outros vinculados à rede de saúde; inovar em políticas e programas de saúde; constituir-se em referência da saúde pública e agregar novos campos do conhecimento ao ensino para fortalecimento da Saúde Pública/Coletiva.

Na década de 1940, Minas Gerais enfrentava surtos de esquistossomose e de ancilostomose (amarelão), de modo que a primeira edição do curso de Saúde Pública foi iniciada em março de 1947, um ano após a criação da Escola, visando à formação e ao aperfeiçoamento de sanitaristas. Contudo, o primeiro curso da ESP-MG foi o de malária, iniciado em fevereiro do mesmo ano.

A formação de profissionais de saúde torna-se mais importante no contexto do estado à medida que a saúde passa a ser reconhecida como importante função do governo, a partir da década de 1950, tornando a qualificação na área fundamental para ingresso na carreira pública nos anos seguintes. A formação de sanitaristas, antes voltadas apenas para médicos amplia-se com a formação de outros profissionais, como as visitadoras sanitárias, iniciada em 1952, profissionais formadas para atuar com orientações e elo entre a população e a política de saúde, considerando os diferentes contextos sociais e sanitários da época. 
As primeiras quatro décadas de atuação da ESP-MG formaram profissionais em diversas áreas: além da formação em saúde pública, destacam-se a nutrição, imunização, administração hospitalar, saúde e segurança no trabalho, saúde escolar, saúde bucal, hanseníase, radiologia e controle de doenças transmissíveis.

A escola tem papel importante na reforma sanitária que deu origem ao Sistema Único de Saúde (SUS) em 1990, e a própria constituição da Saúde Coletiva. Nomes importantes do movimento, como Sérgio Arouca, foram docentes do Curso de Especialização em Saúde Pública. Acompanhou as mudanças das concepções de saúde e desenvolveu projetos para fortalecimento das ações de promoção da saúde.

Ao longo das sete décadas de atuação, mais de 300 mil trabalhadores da saúde pública foram qualificados, abrangendo todo o território de Minas Gerais, em ações presenciais na sede e descentralizadas em todas as regionais do estado e atualmente em cursos na modalidade a distância. Cerca de 9 mil profissionais envolvidos, entre docentes, coordenadores, auxiliares e facilitadores do processo ensino-aprendizagem.

Em 2015, a ESP-MG passa a ser a condutora da Educação Permanente em Saúde (EPS) no Estado. Nesse mesmo ano, a Escola foi citada no Plano Mineiro de Desenvolvimento Integrado (PMDI), ratificando o papel da instituição para formar, qualificar e valorizar os trabalhadores do SUS. Destaque para as especializações em Saúde Pública, Direito Sanitário, Comunicação e Saúde e Gestão Hospitalar.

O curso mais tradicional da ESP-MG, a Especialização em Saúde Pública, recebeu da Agência de Acreditação Pedagógica vinculada à Associação Brasileira de Saúde Coletiva - ABRASCO, em 2017, o certificado de excelência na formação de sanitaristas. Reconhecimento formal da competência da instituição para realizar a formação de profissionais para o SUS.

Em junho de 2019 a Escola completou 73 anos de história e promoveu diversos debates acerca da formação dos profissionais para a saúde pública, sentidos do trabalho no SUS, atuação interprofissional, saúde e ambiente, políticas de drogas e outros. Ao todo, somam-se mais de 700 ações educacionais realizadas em diversas temáticas e linhas de atuação baseadas na Educação Permanente em Saúde (EPS).

\section{A Especialização em Comunicação e Saúde}

Em 2013 a Escola abriu seleção para a primeira turma da Especialização em Comunicação em Saúde. É a única instituição do estado e uma das únicas do país a ofertar o curso na temática e modalidade. A demanda pela Especialização surgiu a partir de conversas da ESP-MG com a Assessoria de Comunicação Social da Secretaria de Estado de Saúde (SES), realizadas em 2012. A questão central era o diagnóstico de falta de conhecimento dos jornalistas e demais profissionais de comunicação acerca do Sistema Único de Saúde, o SUS. Além disso, pensou-se em oferecer uma especialização inspirada no curso similar já existente no Instituto de Comunicação e Informação Científica e Tecnológica em Saúde da Fiocruz, no Rio de Janeiro/RJ.

O atual assessor de comunicação da ESP-MG e coordenador da pós-graduação em Comunicação e Saúde, jornalista Jean Souza, relembra as circunstâncias em que o curso foi idealizado:

Quando ingressei a Escola, no início de 2013, pela minha formação em comunicação e experiência em saúde pública, pediram-me para trabalhar na possibilidade da criação do curso, analisei a demanda e fui buscar o conhecimento sobre o campo. Após ingressar no Mestrado em Informação e Comunicação e Saúde, descobri a amplitude do Campo da Comunicação e Saúde e, a partir daí aquela visão de mídia e saúde foi ampliada para questões comunicacionais que vão além das pautas de saúde dos noticiários, que englobam a comunicação/interação face a face, campanhas institucionais, relações entre profissionais de saúde e usuários, trabalho em equipe, comunicação no SUS, mediação da política pública com cidadão, construção social dos sentidos e discursos da saúde, mobilização social e outros (JS, Belo Horizonte). 
Atualmente na terceira turma, o projeto pedagógico do curso ${ }^{4}$ prevê a oferta de 40 vagas e carga horária de 448h/a, subdivididas em 38oh/a de aulas presenciais, 4oh/a para elaboração do Trabalho de Conclusão de Curso (TCC) e 24h/a para bancas de defesa. Ao todo, o curso tem a duração de um ano e meio, com encontros quinzenais, de quinta-feira a sábado.

Após amplo debate interno e com a SES-MG, os objetivos estabelecidos foram: Objetivo geral: Especializar profissionais que atuam ou desejam atuar em áreas de interface da Comunicação e Saúde. Objetivos específicos: a) Apresentar e discutir o contexto histórico e os princípios que regem a política, planejamento, organização, financiamento, regulação e gestão do Sistema Único de Saúde (SUS), em âmbito estadual e nacional; b) Identificar e discutir as tendências da comunicação na sociedade contemporânea e sua influência na criação/redefinição de valores, construção das políticas públicas e práticas relacionadas com a saúde; c) Desenvolver a capacidade de análise crítica em relação a políticas, métodos, técnicas e tecnologias de comunicação relacionadas à saúde; d) Discutir a importância da comunicação para construção e manutenção das políticas públicas de saúde, ferramenta para a participação social e promoção do vínculo e do cuidado; e) Fomentar as políticas do SUS por meio da comunicação e informação de forma a contribuir para evolução do sistema, participação social e maior adesão pelos usuários e sociedade; f) Contribuir para a consolidação do campo da Comunicação e Saúde.

O processo seletivo da primeira turma ocorreu no final de 2013, com 40 vagas e início das aulas em março de 2014. Jean Souza afirma que a primeira turma foi formada apenas por profissionais da Comunicação, com exceção de uma psicóloga de formação, mas que atuava no Núcleo de Comunicação/ Mobilização da Secretaria Estadual de Saúde. Os alunos eram oriundos de veículos de comunicação e das assessorias de comunicação dos órgãos/instituições de saúde vinculadas ao SUS, Fundações, Municípios, Estado, Hospitais. Os alunos recém-graduados com registro no Ministério do Trabalho também poderiam ser admitidos, uma vez que, conforme explica o coordenador do curso:

...os currículos de jornalismo não tratam das questões de saúde pública, faz-se importante qualificar este profissional já no início da carreira, a fim de que ele possa contribuir com a construção social da saúde como direito. (JS, Belo Horizonte).

Para a segunda turma, no ano seguinte, também com 40 vagas, a coordenação e o corpo docente perceberam a necessidade de abrir o curso para todos os profissionais que atuam na saúde, com reserva de vagas para profissionais de comunicação, o que foi mantido para a $3^{\mathrm{a}}$ turma, 2018-2020. Durante o processo avaliativo da primeira turma, foi constituído um grupo de trabalho. Nas discussões ficou evidenciada a dificuldade dos profissionais de comunicação em conciliar demandas de trabalho com horários de estudo, de modo que seria interessante permitir o ingresso de profissionais de qualquer formação superior atuante no SUS. Constatou-se, na avaliação realizada após o término da segunda turma, que essa foi uma decisão acertada, pois favoreceu a troca e o compartilhamento de experiências entre alunos oriundos de instituições diversas, embora o problema da escassez de tempo da parte dos profissionais de comunicação permanecesse. 
Em relação à grade curricular, foi decisiva a influência do curso da Fiocruz:

O primeiro esboço da Matriz foi feito pelo grupo que discutia o curso antes da minha chegada à ESP$M G$, inspirada no curso de Especialização em Comunicação e Saúde da Fiocruz. Ainda inspirada na Grade da Fiocruz e com a busca de Referências do Campo da Comunicação e Saúde, como o Livro Comunicação e Saúde (ARAÚJO, CARDOSO, 2007), artigos, textos, referências de teorias da comunicação, do jornalismo, assessoria de imprensa e produção de mídias, montamos os conteúdos de comunicação da matriz/ementa. Para compor a grade de disciplinas de saúde pública, em que a escola é referência, buscamos as matrizes e currículos dos cursos de saúde pública e as principais questões que balizam a formação do sanitarista (especialista em saúde pública). Fizemos a junção de temas específicos de saúde que entendemos ser base para que profissionais que nunca tiveram contato com saúde pública pudessem ter uma visão mais ampla e menos generalista e midiática do tema. Somamos a isso os conteúdos de comunicação e os mais específicos do Campo da Comunicação e Saúde. Em todos os conteúdos, pensamos em ementas que fizessem inserções da saúde (disciplinas de comunicação) e da comunicação (disciplinas de saúde pública) (JS, Belo Horizonte).

A matriz curricular foi, então, constituída em sete módulos: Módulo I (Introdução à Saúde Pública e Comunicação e Saúde); Módulo II (SUS: política e gestão); Módulo III (Fundamentos de Comunicação e Implicações na Saúde); Módulo IV (Comunicação e Saúde no Discurso e na Literacia); Módulo V (Práticas da Comunicação e Saúde no SUS); Módulo VI (Produções em Comunicação e Saúde) e Módulo VII (Elaboração e Apresentação de TCC). Cada módulo compreende de uma a quatro disciplinas, entre elas: Comunicação e Literacia em Saúde, Saúde e Sociedade, Redes de Atenção à Saúde, Produção em Comunicação e Saúde e a disciplina objeto deste artigo - Teorias da Comunicação e Contemporaneidade.

\section{Teorias da comunicação, linguagem e intersubjetividade}

A interface entre comunicação e saúde se respalda no pressuposto de que o saber e as práticas de ambas as áreas são constituídos por um aspecto simbólico significativo, conforme nos aponta Teixeira: "saúde, doença e intervenção, praticadas pela economia do contemporâneo, produzem prescrições, sintomas, mortes, tratamentos e curas, mas também uma sensibilidade específica aos modos de produção de vida ou de adoecimento, aos modos de produção coletiva de novas subjetividades. Subjetividade que se dá no contato, na comunicação, no encontro, em que se lançam dispositivos que operam como redes de interfaces" 5 (p. 22).

Diferentes concepções a respeito do que significa ser saudável, o que é qualidade de vida, e o que se espera de um profissional e dos serviços de saúde circulam pelo meio social. Narrativas diversas dão conta daquilo que seria considerado 'normal' e 'patológico', de acordo com saberes tradicionais e com vivências cotidianas. O conhecimento científico, de algum modo, tenta se impor sobre o senso comum e, em grande parte, as narrativas coexistem e disputam lugar no imaginário coletivo.

De acordo com a citação acima, percebemos a importância da linguagem concebida como intersubjetividade para a construção do conhecimento a respeito da saúde. Os profissionais, tanto da Comunicação quanto da Saúde, experimentam em seu cotidiano profissional essas tensões, de modo que conhecer os fundamentos da comunicação humana (para os profissionais da saúde) ou aprofundá-los (para os profissionais da comunicação) torna-se imperativo.

No curso de especialização em Comunicação e Saúde Pública da ESP-MG, a disciplina Teorias da Comunicação é a primeira a ser ministrada no terceiro módulo. O aluno, neste estágio, já cursou pelo menos seis meses da pós-graduação; prevendo que esteja familiarizado com conceitos mais básicos das primeiras disciplinas. Com carga horária de 4oh/a, a ementa prevê dois momentos: 1) Conceitos de comunicação, 
principais correntes teóricas, meios de comunicação, mídia e mediações. A centralidade da mídia nas sociedades contemporâneas; 2) Principais características da globalização; sociedade da informação (ou do conhecimento); convergência tecnológica; alterações no mercado de comunicação. Estado e políticas públicas de comunicação no Brasil; o cenário atual; novas tendências de políticas de comunicação. Responsabilidade social da comunicação e imprensa e suas relações com a saúde pública.

No primeiro momento, a ementa não difere daquelas encontradas nos cursos de comunicação social, tanto em nível de graduação ou de pós-graduação. No curso em tela, é importante lembrar que as turmas são híbridas, o que demanda do professor tanto um esforço didático - para não ser repetitivo para uma parte da turma e incompreensível para a outra - quanto motivacional - para despertar o interesse a respeito do assunto e mantê-lo durante as aulas.

As aulas iniciais, portanto, são cruciais para que os alunos compreendam que a) a comunicação humana pode ser objeto de reflexão e investigação científica; b) existe uma disciplina que se propõe a fazê-lo; c) a disciplina é formada por diversas correntes de pensamento que buscam compreender o fenômeno, e que as diversas abordagens podem ser conflitantes entre si; d) a comunicação não é 'produto' ou 'resultado', mas um processo de construção de intersubjetividade, envolvendo contexto, interlocutores e diferentes linguagens; e) essa disciplina visa a construir um olhar comunicacional, que não está restrito somente às mídias, por mais 'vistosas' que elas sejam na contemporaneidade; f) a comunicação faz interface com as questões de saúde pública, uma vez que essas são também questões construídas pela linguagem e pelas interações sociais.

Na terceira turma, foi solicitado, a título de exercício, que os alunos fizessem uma autorreflexão, escrevendo um texto breve a respeito de seu cotidiano comunicacional, relatando seus hábitos e preferências durante um dia normal. Como esta turma era mista (composta por comunicadores e por profissionais de saúde), o objetivo foi que ambos comparassem sua capacidade de percepção do papel da comunicação em suas vidas. As redações foram lidas em voz alta para a turma, ensejando uma discussão sobre o (possível) excesso de consumo e a baixa capacidade crítica do público, incluindo os próprios alunos.

O segundo momento se inicia com uma proposta que inter-relaciona comunicação e política, de modo a situar os meios de comunicação e a imprensa no contexto mercadológico global. Em seguida, faz-se uma discussão concernente à responsabilização midiática. É nessa hora que termos como mobilização, extensão e diálogo surgem e se tornam essenciais para a construção da interface entre Comunicação e Saúde. 


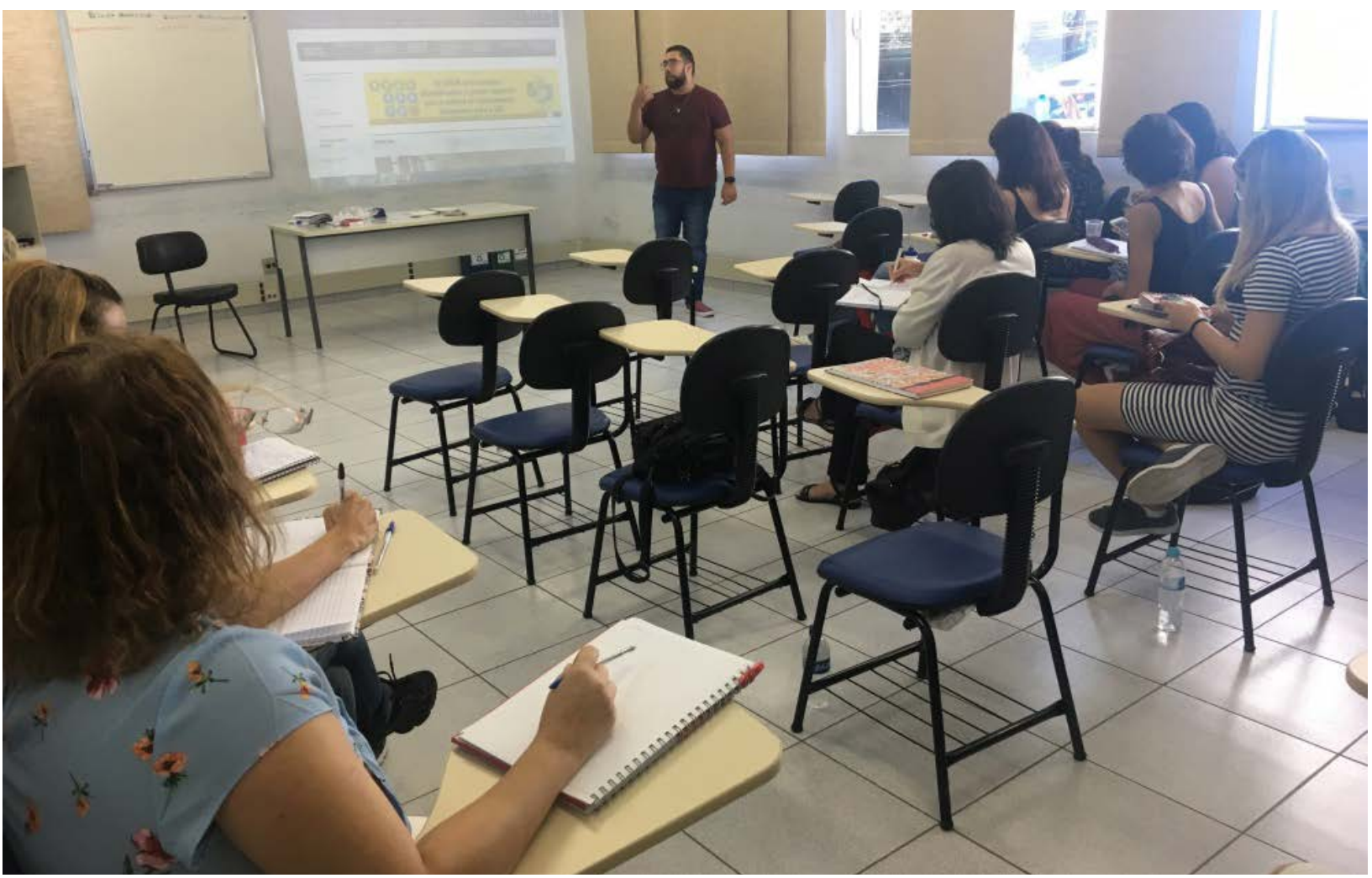

Figura 1 - Terceira turma do curso de pós-graduação em Comunicação e Saúde

Fonte: Acervo da coordenação do curso Comunicação e Saúde (ESP-MG) (2019).

\section{Mobilização, extensão ou diálogo?}

Simeone afirma que o termo 'mobilização', quando aplicado ao campo da epidemiologia, tradicionalmente se refere a um conjunto de esforços para debelar uma situação momentânea de risco, "carrega o significado de uma movimentação intensiva para debelar fatores que concorrem para a expansão de epidemias e prevenir a ocorrência de novos casos de determinadas doenças"'.

A comunicação, nessas circunstâncias, é utilizada como mais um recurso de combate, adquirindo um caráter instrumental para uma situação fora de controle. Nesses casos, o termo 'campanha', também uma metáfora bélica, é utilizado de forma a atingir o maior número de pessoas no menor espaço de tempo com mensagem simples e direta, de forma a obter resultados rápidos e eficazes.

Simeone $^{6}$ nos alerta, porém, que na complexidade das sociedades globalizadas, as situações antes tidas como excepcionais agora são permanentes, de modo que o risco embutido nelas é estendido para a vida cotidiana. A ordem é que o indivíduo não se descuide nem por um minuto, exercendo vigilância e cuidado nos mais ínfimos aspectos de sua saúde. Essa ampliação da noção de risco e sua representação nas narrativas midiáticas é um dos temas discutidos na disciplina Teorias da Comunicação.

Durante as aulas, procurou-se mostrar que há certamente um momento em que a comunicação em saúde deve ser emergencial, como na ocorrência de surtos e epidemias. O trabalho de comunicação, no entanto, tem um caráter bem mais de médio e longo prazo: não é difícil perceber que o entendimento ampliado de mobilização social requer uma mudança nas formas como a comunicação é compreendida e sobre as funções que assume, muito diferentes de uma prática de difusão e de propaganda e mais voltadas para estabelecer interlocuções e gerar e manter vínculos. Há nessa visão uma clara aposta em estratégias de comunicação que consigam a um só tempo posicionar amplamente os múltiplos riscos em ambiente de insegurança e tecer relações relativamente estáveis em meio às contínuas ameaças - o que está implícito nas ideias de coesão e de continuidade ${ }^{6}$ (p. o3). 
"Manter a interlocução" e "gerar vínculos", como preconiza o autor ${ }^{6}$, demandam uma mudança de postura tanto do comunicador quanto do profissional da saúde, que precisam 'descer do pedestal' de seus conhecimentos estabelecidos/especializados e se abrir para o entendimento da alteridade. Não se cria vínculo desprezando o saber do outro, não se cria vínculo em curto prazo.

Para o educador Paulo Freire, essa atitude de desprezo seria típica de uma concepção extensionista, a qual não leva em conta o conhecimento prévio e a experiência dos interlocutores no processo de comunicação, de forma que se torne necessário transmitir algo a quem não sabe nada. Quando se reduz esta interface a um conjunto estrito de práticas realizadas por profissionais universitários, despreza-se o saber popular, o sentido dado às práticas de saúde no cotidiano, bem como a contribuição de práticas ditas 'alternativas'. Nessa visão, o comunicador se posiciona 'acima' do público, como lembrou Freire: “(...) na medida em que, no termo extensão, está implícita a ação de levar, de transferir, de entregar, de depositar algo em alguém, ressalta, nele, uma conotação indiscutivelmente mecanicista. Mas, como este algo que está sendo levado, transmitido, transferido [...] é um conjunto de procedimentos técnicos, que implicam um conhecimento, que são conhecimento, se impõem as perguntas: será o ato de conhecer aquele através do qual um sujeito, transformado em objeto, recebe pacientemente o conteúdo do outro? Pode este conteúdo, que é conhecimento de ser tratado como algo estático? Estará ou não submetendo o conhecimento a condicionamentos histórico-sociológicos?”7 (p. 26).

A essa visão extensionista contrapõe-se uma visão dialógica, a qual ampara a disciplina Teorias da Comunicação do curso de especialização em Comunicação e Saúde da ESP-MG. Na visão dialógica, comunicação é concebida como intrínseca ao processo de mobilização, e não exterior a ele. Quando se menciona o diálogo, o que está em jogo é a capacidade de reconhecer de antemão a alteridade e considerá-la como interlocutor legítimo. A comunicação dialógica, portanto, seria aquela assentada na horizontalidade, na qual os interlocutores não se sobrepõem em virtude de uma hierarquia dada por antecipação.

É uma concepção na qual comunicação e educação são indissociáveis, porque são diferentes dimensões/ momentos de um mesmo processo de construção. Na interface Comunicação e Saúde, os meios e processos de comunicação são vistos como agentes sociais de saúde. Nessa perspectiva, os profissionais, ao elaborarem representações midiáticas da saúde pública, devem ter em mente que o direito à saúde, previsto na CF de 1988, somente se efetivará a partir de um conceito de saúde integral, em que o acesso seja o mais universal possível e que contextos sejam considerados (equidade).

Outro aspecto a se considerar, para além do acesso à informação, é a pluralidade de vozes: "O direito à comunicação abrange não só o acesso às informações como também a (falta de) expressão dos cidadãos, das instituições e dos grupos sociais, inclusive para explicitar suas necessidades de saúde. Como conceito, deveria servir como referência e contraponto à falta de diversidade e pluralidade que caracteriza os meios de comunicação brasileiros, diversificando positivamente a escolha dos temas, as abordagens e as vozes selecionadas para falar sobre a saúde no país”8 (p. 14).

O que se espera, com a inserção da disciplina Teorias da Comunicação, é que o aluno profissional de Comunicação aprofunde seus conhecimentos, ao mesmo tempo em que amplie a perspectiva de atuação, buscando atuar não somente nas emergências, mas principalmente na construção cotidiana da saúde. Para os alunos profissionais de saúde, espera-se que eles compreendam que a promoção da saúde deve ser alcançada por meio do diálogo, de forma que o conhecimento das diferenças maneiras de mediação é essencial para o seu trabalho. Em uma turma mista, como foram a segunda e a terceira, o intercâmbio de saberes é fundamental. A turma, na prática, já exercita a comunicação e reflete a seu respeito coletivamente.

Dessa forma, entende-se que estudar as Teorias da Comunicação, em um curso de especialização para comunicadores e profissionais de saúde, tem muito a contribuir para a formação e o aperfeiçoamento deles, não somente levando ao conhecimento temas de investigação e conceitos técnicos, mas principalmente por se constituir em uma disciplina durante a qual a reflexão, o debate e a construção de uma interface se fazem presentes. É um momento único de construção coletiva. 


\section{Referências}

1. Braga JL. A constituição do campo da comunicação. In: Cohn G., Braga JL, Martino LC, Lopes MIV, Sodré M, Samain E. Campo da comunicação: caracterização, problematizações e perspectivas. João Pessoa: Universitária; 2001. p. 11-39.

2. Araújo IS, Cardoso JM. Comunicação e Saúde. Rio de Janeiro: Fiocruz; 2007.

3. Miranda $H$, Heringer $L$, Caiafa $R$, organizadores. ESP-MG: tradição em inovar: 68 anos de história. Belo Horizonte: ESP-MG; 2014.

4. Escola de Saúde Pública (MG). Projeto Pedagógico do curso de Pós-graduação em Comunicação e Saúde. Belo Horizonte: ESP-MG; 2013.

5. Teixeira RR, Cyrino AP. As ciências sociais, a comunicação e a saúde. Ciênc Saúde Coletiva [Internet]. 2003 [citado em 2020 jan. 18];8(1):151-72. Disponível em: https://bit.ly/3b0noXG.

6. Simeone MH. Comunicação e mobilização em saúde. Diversa [Internet]. 2008 nov. [citado em 2020 jan. 18];7(16). Disponível em: https://bit.ly/3e5KqxY.

7. Freire P. Extensão ou comunicação? São Paulo: Paz e Terra; 2010. p.49.

8. Emerich TB, Cavaca AG, Gentilli V, Emmerich A. Necessidades da saúde e direito à comunicação em tempos de mediação. Rev Eletron Comun Inf Inov Saúde [Internet]. 2016 out.-dez. [citado em 2020 jan. 18];10(4). Disponível em: https://bit.ly/3c3lmWF. 